\title{
Lens fibers have a fully functional ubiquitin-proteasome pathway
}

\author{
Paulo Pereira $^{\mathrm{a}, \mathrm{b}}$, Fu Shang ${ }^{\mathrm{a}, *}$, Marisa Hobbs ${ }^{\mathrm{a}}$, Henrique Girão ${ }^{\mathrm{a}, \mathrm{b}}$, Allen Taylor ${ }^{\mathrm{a}}$ \\ ${ }^{a}$ Laboratory for Nutrition and Vision Research, USDA HNRC at Tufts University, 711 Washington Street, Boston, MA 02111, USA \\ ${ }^{\mathrm{b}}$ Centre of Ophthalmology, Biomedical Institute for Research in Light and Image (IBILI), Faculty of Medicine, \\ University of Coimbra, 3000 Coimbra, Portugal
}

Received 23 August 2002; accepted in revised form 12 December 2002

\begin{abstract}
We previously showed that lens epithelial cells have a fully functional ubiquitin-proteasome pathway (UPP) and that ubiquitin-conjugating activity is up-regulated in response to oxidative stress. In this study we assessed the protein levels and activities of different components of the UPP in lens fibers. Calf lenses were dissected into four different regions: epithelial layer, outer cortex, inner cortex and nucleus. Relative levels of ubiquitin-activating enzyme (E1), ubiquitin-conjugating enzymes (E2s), endogenous ubiquitin conjugates, $19 \mathrm{~S}$ and $20 \mathrm{~S}$ proteasome subunits were determined by Western blotting. The activities of E1 and E2 were determined by thiol ester assays and the activities of the proteasome and isopeptidases were determined using ubiquitinated $\alpha$-lactalbumin as a substrate. This work demonstrates that lens fibers, including those in the nuclear region, contain most, if not all, of the components for the UPP. Ubiquitin conjugation activity, proteasome activity and isopeptidase activity were also detected in all layers of the lens. The reduced ubiquitin conjugation activity in the inner regions of the lens appeared to be due to a decline in levels of a specific family of E2s, Ubc4 or Ubc5, which were shown to be the rate-limiting enzymes for the formation of high mass conjugates in the lens. Supplementation of Ubc4 or Ubc5 can partially restore the ubiquitin conjugation activity in the inner regions of the lens. Since Ubc4 and Ubc5 are involved in selectively ubiquitinating damaged or abnormal proteins, the decline in levels and activities of these E2s may be responsible for the accumulation of abnormal proteins in inner regions of the lens.
\end{abstract}

(C) 2003 Published by Elsevier Science Ltd.

Keywords: differentiation; development; lens; proteasome; ubiquitin

\section{Introduction}

The ubiquitin-proteasome pathway (UPP) is a major cytosolic proteolytic pathway in most eukaryotic cells. There are two stages in the UPP: substrate-recognition by formation of ubiquitin-protein conjugates and the subsequent degradation of the ubiquitin conjugates by the $26 \mathrm{~S}$ proteasome. In its simplest form, ubiquitination involves the attachment of multiple molecules of ubiquitin to protein substrates (Hershko and Ciechanover, 1998). In order to initiate this process, ubiquitin is activated by the formation of a high-energy thiol ester with ubiquitinactivating enzyme (E1), the first enzyme in the ubiquitination pathway. The ubiquitin is then transferred to one of many ubiquitin-conjugating enzymes (Ubc or E2s), also via

\footnotetext{
* Corresponding author. Dr Fu Shang, Laboratory for Nutrition and Vision Research, USDA HNRC at Tufts University, 711 Washington Street, Boston, MA 02111, USA.

E-mail address: fu.shang@tufts.edu (F. Shang).
}

formation of a thiol ester. Subsequently, ubiquitin is transferred directly to substrates or is transferred to substrates via one of several ubiquitin ligases (E3s). Multiple isoforms of E2s and E3 have been identified in each species. The multiplicity of E2 and E3 enzymes is responsible for the substrate specificity of the UPP. Multiple ubiquitin moieties attach to substrate proteins to form ubiquitin chains. Thus, most of the ubiquitin conjugates attain high masses. These ubiquitin conjugates are generally recognized and degraded by the $26 \mathrm{~S}$ proteasome at rates which are generally proportional to rates of ubiquitin-protein conjugate formation (Chin et al., 1982; Obin et al., 1994). The $26 \mathrm{~S}$ proteasome consists of two large subcomplexes, the $20 \mathrm{~S}$ particle with a barrel-shaped structure, which carries the catalytic activity, and the $19 \mathrm{~S}$ regulatory particle, which caps both ends of the barrel (Baumeister et al., 1998). The 19S particle contains ATPase activity and is presumably responsible for the recognition of the ubiquitinated proteins (Tanaka and Tsurumi, 1997; 
Lam et al., 2002). In some instances, ubiquitin-protein conjugates are deconjugated by isopeptidases (Wilkinson et al., 1995; Lam et al., 1997), rather than being degraded by the $26 \mathrm{~S}$ proteasome. Therefore, ubiquitination may have other functions in addition to targeting proteins for degradation. However, non-proteolytic functions for ubiquitination remain to be elucidated.

The lens is composed of two types of cells: epithelial cells and fiber cells. Epithelial cells cover the anterior surface of the lens and fiber cells occupy almost the entire volume of the lens. The fiber cells are differentiated from the epithelial cells. During differentiation, lens epithelial cells exit from cell cycle (Chamberlain and McAvoy, 1987) and undergo significant morphological and biochemical changes that result in the formation of fully differentiated fiber cells, where virtually all organelles, including the nuclei, are absent. This unique pattern of differentiation occurs in the equatorial region of the lens, and fibers with increasingly advanced stages of differentiation accumulate concentrically at the interior of the lens (Kuszak, 1995). Proper execution of the differentiation program and formation of mature fibers seem to be required for lens transparency since abnormalities that result in incomplete degradation of intracellular organelles are associated with various forms of cataract (Pan and Griep, 1994; Nakamura et al., 1995).

A fully functional UPP has been demonstrated in the lens epithelial cell (Huang et al., 1993, 1995; Shang and Taylor, 1995; Shang et al., 1997a, 1999). The ubiquitin conjugating activity and proteolytic activity in lens epithelial cells are up-regulated during recovery from oxidative stress (Shang et al., 1997b). One of the functions for the UPP is to selectively degrade damaged proteins (Shang et al., 2001). To test our hypothesis that the UPP plays a role in selective removal of damaged proteins from the lens, it is necessary to determine if the lens fibers also have a fully functional UPP, because the majority of damaged proteins are in fiber cells. We report here that the UPP components examined in this work were detected in all layers of the lens, including fibers in the nuclear region. However, the protein levels and activities of these components for the UPP decreased in inner regions of the lens. The ubiquitin conjugation activity in the lens is rate limited by Ubc $4 / 5$, a family of E2s that are involved in degradation of damaged or obsolete proteins (Seufert and Jentsch, 1990). Supplementation of Ubc4 or Ubc5 partially restored the conjugation activities in fibers in the inner regions of the lens. Taken together, the data demonstrate that lens fiber cells, including those in the nucleus, have a fully functional UPP.

\section{Materials and methods}

\subsection{Chemicals}

Unless otherwise specified all chemicals used were from Sigma (St Louis, MO, USA) or Fisher Scientific company
(Fairlawn, NJ, USA). Acrylamide $N, N^{\prime}$-methylene-bisacrylamide, $N, N, N^{\prime}, N^{\prime}$-tetramethylenediamine, 2-mercaptoethanol, sodium dodecyl sulfate (SDS), glycine and protein molecular mass standards were obtained from Bio-Rad (Richmond, CA, USA). $\mathrm{Na}^{125} \mathrm{I}$ and ${ }^{125}$ I-protein A were obtained from Du Pont/NEN (Boston, MA, USA). Ubiquitin aldehyde (Ubal), recombinant Ubc2, Ubc3, Ubc5, Ubc7 and Ubc10 were purchased from Boston Biochem (Cambridge, MA, USA). Recombinant Ubc1 was a gift from Dr C.M. Pickart and recombinant Ubc5 was provided by $\mathrm{Dr}$ S. Wing. MG132 ( $N$-carbobenzoxyl-L-leucinylL-leucinal) was obtained from Calbiochem (San Diego, CA, USA). Sepharose-protein A was purchased from Amersham Pharmacia Biotech (Uppsala, Sweden).

\subsection{Antibodies}

The monoclonal antibody to ubiquitin conjugates (FK2) was a kind gift from Dr Takada and polyclonal antibodies to ubiquitin were produced in New Zealand White rabbits by injection of SDS-denatured ubiquitin conjugated to $\gamma$-globulin. Antibodies to Ubc2, Ubc5 and Ubc7 were purchased from Boston Biochem (Cambridge, MA, USA). Antibody to E1 was prepared by immunizing rabbit with synthetic E1 peptide conjugated to ovalbumin (Shang et al., 2001). Antibody to subunit 7 of the 19 S proteasome particle was a kind gift from Dr Tanaka, which was produced in rabbit using recombinant MMS1 (S7) as the antigen. The antibody to subunit $\alpha 4$ of the 20S proteasome was purchased from Affiniti Research Products (Exeter, UK).

\subsection{Lenses}

Calf and cow eyes were obtained from the local slaughterhouse and the lenses were extracted on ice. The anterior capsule with attached epithelial cell layer was carefully peeled off and designated as the epithelial layer. The outer layers of the lens (about one third of the lens volume) were carefully peeled off and designated as the outer cortex. Then the middle layers (about one third of the lens volume) were isolated in a similar way and designated as the inner cortex. The remaining material (about one third of the lens mass) was designated as the nucleus. These lens regions were pooled from 8 to 10 lenses and immediately stored at $-80^{\circ} \mathrm{C}$ until use.

\subsection{Protein electrophoresis and immunoblotting}

Different layers of the lens were homogenized in $50 \mathrm{~mm}$ Tris-HCl buffer, $\mathrm{pH} 7 \cdot 6$, containing $5 \mathrm{~mm}$ EDTA, $1 \% \mathrm{NP}-$ $40,0 \cdot 1 \%$ SDS, $10 \mathrm{~mm}$ NEM and $2 \mathrm{~mm}$ AEBSF. The homogenates were centrifuged at $12000 \mathrm{~g}$ at $4^{\circ} \mathrm{C}$ for $10 \mathrm{~min}$ and protein concentrations in the supernatants were determined by the Lowry method. For immunoblotting, supernatants of different regions of the lens were mixed with an equal volume of $2 \times$ Laemmli buffer, boiled for $5 \mathrm{~min}$, 
loaded on a $12 \%$ polyacrylamide gel and separated by SDS-PAGE. Ten micrograms of epithelial layer proteins and $100 \mu \mathrm{g}$ of fiber proteins were loaded in respective lanes of the gel. After electrophoretic separation, proteins were transferred to nitrocellulose with a Bio-Rad sandwich transfer system. The blots were probed with the appropriate primary antibodies as described in the figure legends. Specifically bound antibodies were detected by secondary antibodies and visualized using the SuperSignal kit from Pierce (Rockford, IL, USA).

\subsection{Activity of ubiquitin-activating and ubiquitin-conjugating enzymes}

Activities of the ubiquitin-activating enzyme (E1) and ubiquitin-conjugating enzymes (E2s) were determined by their ability to form thiol esters with ubiquitin (Ciechanover et al., 1982; Hershko et al., 1983; Shang et al., 1997a,b). Different regions of the lens or harvested epithelial cells were homogenized in $50 \mathrm{~mm}$ Tris- $\mathrm{HCl}, \mathrm{pH} 7.6$ and $1 \mathrm{~mm}$ DTT on ice. Homogenates were then centrifuged at $12000 \mathrm{~g}$ for $20 \mathrm{~min}$ at $4^{\circ} \mathrm{C}$. Thiol ester reactions contained (final concentration) $50 \mathrm{~mm}$ Tris $-\mathrm{HCl}, \mathrm{pH} 7 \cdot 6,5 \mathrm{~mm}$ $\mathrm{MgCl}_{2}, 1 \mathrm{~mm}$ DTT, $1 \mathrm{~mm}$ ATP, $10 \mathrm{~mm}$ creatine phosphate, $5 \mu \mathrm{g}$ creatine phosphokinase, $2 \mu \mathrm{g}{ }^{125}$ I-ubiquitin $\left(\sim 10^{6} \mathrm{cpm}\right)$ and $500 \mu \mathrm{g}$ of lens fiber supernatant or $50 \mu \mathrm{g}$ of lens epithelial cell supernatant. The reaction mixture was incubated for $5 \mathrm{~min}$ at $37^{\circ} \mathrm{C}$ to allow formation of thiol esters between ${ }^{125}$ I-ubiquitin and E1 and E2s. The reaction was stopped by addition of an equal volume of either $2 \times$ Laemmli buffer (containing 2-mercaptoethanol) or thiol ester assay buffer (50 mм Tris, 4\% SDS, $8 \mathrm{~m}$ urea, $10 \%$ glycerol, $\mathrm{pH}$ 6.8). After standing at room temperature for $20 \mathrm{~min}, 20 \mu \mathrm{l}$ of sample $(\sim 50000 \mathrm{cpm})$ were loaded on a $12 \%$ gel and proteins were separated by SDS-PAGE. The gel was subsequently dried and exposed to film. Activities of E1 and E2s were determined by quantifying the densities of the respective thiol ester bands on the autoradiogram.

\subsection{Determination of ubiquitin conjugation activity}

The ability of lens supernatants to form de novo ${ }^{125}$ I-ubiquitin conjugates was determined as described above for the thiol ester assays with the following alterations: to inhibit the degradation and deconjugation of the de novo formed ubiquitin conjugates, proteasome activity was inhibited by addition of $80 \mu \mathrm{M}$ MG132 and the isopeptidase activity was inhibited by $8 \mu \mathrm{M}$ Ubal. The reaction proceeded for $20 \mathrm{~min}$ at $37^{\circ} \mathrm{C}$ and was terminated by incubating the reaction mixture with Laemmli buffer at room temperature for $20 \mathrm{~min}$. To test the effect of different E2s on conjugation activity, $100 \mathrm{ng}$ of the respective E2 was added to the conjugation mixture. The ubiquitin-conjugation activity was assessed by quantifying the densities of the bands which represent the high mass ubiquitin conjugates on the autoradiograms.

\subsection{Immunoprecipitation}

Twenty microliters of a 50\% Sepharose-protein A slurry was incubated with $20 \mu \mathrm{l}$ of anti-Ubc 4 or anti-Ubc2 serum for $3 \mathrm{hr}$ at $4^{\circ} \mathrm{C}$ and the mixtures were briefly centrifuged at $12000 \mathrm{~g}$. The supernatants were discarded and the pellets were washed three times with PBS. The final pellets containing the Sepharose-protein A-antibody complexes were incubated with $500 \mu \mathrm{g}$ lens supernatants at $4^{\circ} \mathrm{C}$ overnight. The mixtures were then briefly centrifuged at $12000 \mathrm{~g}$ and the supernatants were tested for conjugation activity. A negative control was produced by carrying out the same procedure with a pre-immune serum.

\subsection{Activity of the proteasome}

To detect the activities of the $26 \mathrm{~S}$ proteasome, $\alpha$-lactalbumin was labeled with ${ }^{125} \mathrm{I}$ and ubiquitin conjugates of the ${ }^{125}$ I-labeled substrate were formed in proteasome-free fraction II of rabbit reticulocytes. To obtain proteasome-free fraction II, the rabbit reticulocyte fraction II was prepared as described (Huang et al., 1993) and then the proteasome was removed from the fraction II by centrifuging at $100000 \mathrm{~g}$ for $5 \mathrm{hr}$ (Gaczynska et al., 1996). The ubiquitin-conjugates of ${ }^{125}$ I-labeled $\alpha$-lactalbumin were isolated chromatographically using a DE52 column and were used as substrates to determine the proteasome activity in the supernatants of each layer of lens fibers. The substrates were mixed with supernatants from each layer of the calf lens in the presence or absence of MG132 and incubated at $37^{\circ} \mathrm{C}$ for $1 \mathrm{hr}$. Degradation rates were determined as the percentage of the substrates which became TCA-soluble after incubation with lens supernatants. The portion of the degradation which was inhibited by MG132 was designated as proteasomedependent degradation and the rest was designated as proteasome-independent degradation.

\subsection{Activity of isopeptidase}

To determine isopeptidase activity, $\alpha$-lactalbumin was labeled with ${ }^{125} \mathrm{I}$, ubiquitinated and isolated as described above. The isolated ubiquitin conjugates were incubated with supernatants of lens fibers and incubated at $37^{\circ} \mathrm{C}$ for 15-60 min in the presence or absence of $4 \mu \mathrm{M}$ Ubal. For a negative control the isolated ubiqutin conjugates of ${ }^{125} \mathrm{I}$ - $\alpha$-lactalbumin were incubated at $37^{\circ} \mathrm{C}$ for $60 \mathrm{~min}$ alone and then mixed with lens fiber supernatant just prior to SDS-PAGE separation. The isopeptidase activity was assessed by the amount of ubiquitin conjugates of ${ }^{125}$ I-labeled $\alpha$-lactalbumin which could be stabilized by Ubal. 


\section{Results}

\subsection{Lens fibers contain all of the components of the UPP}

In order to determine the distribution of the components of the UPP in the lens, calf lenses were dissected into four different regions: 1) the anterior epithelial layer, 2) the outer cortex, 3) the inner cortex, and 4) the nucleus. All regions of the lens contain high mass ubiquitin conjugates (Fig. 1(A)). Levels of the high mass ubiquitin conjugates were highest in the epithelial layer and decreased toward the inner nuclear region. The gradient is even more readily appreciated given that the amount of protein loaded on lane 1 was only one tenth of that loaded on lanes $2-4$. The high levels of ubiquitin conjugates in epithelial and outer cortex layers are likely to reflect the availability of substrates as well as greater catalytic activities that are required for ubiquitination.

Levels of endogenous ubiquitin conjugates depend on the rates of ubiquitination and the rates of degradation of the conjugates by the proteasome or deconjugation by isopeptidases (Chin et al., 1982; Obin et al., 1994; Wilkinson et al., 1995). To determine the mechanism for the decreased level of ubiquitin conjugates in inner layers of the lens, we determined the levels of E1, some E2s and



Fig. 1. Levels of components of the ubiquitin-proteasome pathway in lens fibers. Calf lenses were dissected into epithelial layer (EL), outer cortex $(\mathrm{OC})$, inner cortex (IC), and nucleus $(\mathrm{N})$ regions. Proteins from each region were separated by SDS-PAGE in a $12 \%$ polyacrylamide gel, transferred to nitrocellulose and probed with antibodies to ubiquitin conjugates (A), E1 (B), Ubc2 (C), Ubc5 (D), Ubc7 (E), MMS1 (a subunit of the regulatory PA700 complex of the $26 \mathrm{~S}$ proteasome) (F), and subunit $\alpha 4$ (a subunit of the catalytic core of the $26 \mathrm{~S}$ proteasome) $(\mathrm{G}) .10 \mu \mathrm{g}$ of protein were loaded on lane 1 and $100 \mu \mathrm{g}$ of protein were loaded on lanes $2-4$. The data represent at least three experiments. proteasome subunits in each layer of the lens. Western blot analysis showed that E1 was present in all regions of the lens (Fig. 1(B)). Consistent with previous observations in lens epithelial cells (Shang et al., 1997b), E1 is present in two isoforms in lens tissue with molecular weights of 110 and $117 \mathrm{kDa}$, respectively. The epithelial layer contained the highest amount of E1 and levels of the two isoforms were comparable in the epithelial layer. Both isoforms of E1 decreased toward the inner region of the lens tissue, but levels of the $117 \mathrm{kDa}(\mathrm{E} 1 \mathrm{~A})$ isoform decreased more than the $110 \mathrm{kDa}(\mathrm{E} 1 \mathrm{~B})$ isoform did.

In contrast to the gradual decrease in levels of E1 from the epithelial layer to the nucleus, there was a dramatic decline in levels of E2s (Ubc2 and Ubc7) from the epithelial layer to the outer cortex (Fig. 1(C) and (E)). Considering that the protein load for the epithelial layer is only one tenth of that for fiber cells, the relative concentrations of Ubc2 and Ubc7 in fiber cells were only about $5 \%$ of those in the epithelial layer. However, there were no significant differences in levels of $\mathrm{Ubc} 2$ and $\mathrm{Ubc} 7$ between the outer cortex and the nucleus of the lens. Ubc4 and Ubc5 are two closely related E2s. They are not separable by SDS-PAGE, nor are they distinguishable by the antibody used. Therefore, the immunoreactive band was designated as Ubc4/5. Similar to changes of endogenous ubiquitin conjugates, levels of Ubc4/5 in the outer cortex were lower than those in the epithelial layer (after correction for protein load), but were significantly higher than those in the inner cortex and the nucleus (Fig. 1(D)). In fact, Ubc4/5 were barely detectable in the nucleus of the lens (Fig. 1(D)).

The 26S proteasome in each layer of the lens was assessed by quantifying levels of a representative subunit of the $19 \mathrm{~S}$ regulatory complex and a representative subunit of the 20S catalytic core. Western blotting showed that MMS1 (subunit S7 of the 19S regulatory complex) was present in all layers (Fig. 1(F)). Since there was 10-fold higher protein load on lanes $2-4$ vs lane 1 , it is clear that the relative concentration of this proteasome subunit was markedly higher in the epithelial layer than in fiber cells. Levels of this proteasome subunit decreased progressively between the outer cortex and the nucleus. A similar pattern was shown for levels of the $20 \mathrm{~S}$ core subunits. In the epithelial layer, antibodies to the subunit $\alpha 4$ of the $20 \mathrm{~S}$ core reacted with only one $30 \mathrm{kDa}$ band. The same band was also detected in all layers of fibers, but the density of this band decreased toward the inner nuclear region (Fig. 1(G)). In fibers, a second band with smaller size was also recognized by this antibody (Fig. $1(\mathrm{G})$. The density of the lower band was inversely related to the density of the subunit $\alpha 4$. Thus, it appears that the lower band is a protease cleavage product of the subunit $\alpha 4$. Taken together, these data show that most, if not all, of the components of the UPP are present in fibers of all developmental layers of the lens, although levels of these components decreased in the inner regions of the lens. 


\subsection{Lens fibers retain the ubiquitin conjugation activity}

After demonstration that most of the components of the UPP are present in lens fibers, we determined the activities of the UPP. As noted in the upper panel of Fig. 2, all layers of the lens had the ability to form de novo ubiquitin conjugates using ${ }^{125}$ I-labeled ubiquitin. The most prominent conjugates are of high mass and are retained at the top of the gel (Fig. 2, upper panel). Consistent with the distribution of endogenous ubiquitin conjugates, the epithelial layer and the outer cortex showed the highest conjugation activity and the lens nucleus had the lowest ubiquitin conjugation activity (Fig. 2, upper panel). As judged by the levels of E1-ubiquitin thiol ester, the activity of E1 was also highest in the epithelial layer and lowest in the nucleus (Fig. 2, lower panel). Comparison of E1 protein levels (Fig. 1(B)) with E1 activity (Fig. 2, lower panel) in each layer of the lens revealed that E1 activity decreased faster than E1 protein levels did from the lens epithelial layer to the nucleus of the lens. This suggests that inactivation of the enzyme precedes diminished immunoreactivity. Thiol ester assays also showed that activities of E2s were highest in the epithelial layer and lowest in the nuclear region (data not shown). Taken together, the thiol ester assays and conjugation assays show that enzymes involved in ubiquitin conjugation remain active in differentiated lens fibers, although the specific activities of these enzymes were lower, particularly in the inner regions of the lens.

The coincidental decrease of E1 and E2 activities in inner layers of the lens suggests that the diminished conjugation activity in these regions is caused by the decline in E1 and/or E2 activities. To test which of these enzymes contribute to the decreased conjugation activity in inner regions of the lens, E1 or selected E2s were supplemented to the supernatants of lens fibers and the conjugation activity was tested. Supplementation of supernatants of inner regions of the calf lens with purified E1 resulted in only a marginal increase in the conjugation activity, even when

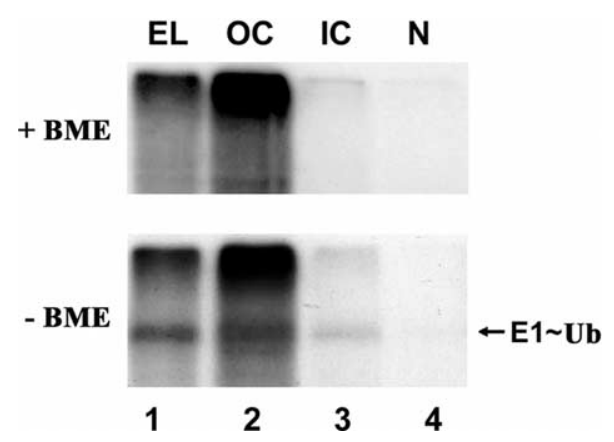

Fig. 2. All developmental layers of the lens have the capability to conjugate ubiquitin to endogenous proteins. Calf lenses were dissected into epithelial layer (EL), outer cortex (OC), inner cortex (IC) and nucleus (N) regions. The ubiquitin conjugation activity was determined by the capacity to conjugate ${ }^{125}$ I-labeled ubiquitin to endogenous substrates (upper panel). The activity of E1 was determined by levels of E1-ubiquitin thiol ester (lower panel). The data represent three experiments. the activity of E1 added to fibers (as measured by thiol ester formation) was comparable to the activity of endogenous E1 in the epithelial layer (data not shown). This suggests that the decreased E1 activity is not the major factor for the decreased ubiquitin conjugation activity in the inner regions of the lens. Similarly, supplementation of lens fiber supernatants with Ubc1, Ubc2, Ubc3, Ubc7 and Ubc10 had little effect on the ubiquitin conjugation activity (Fig. 3). The apparent increase in ubiquitin conjugation in lane 3 of Fig. 3(B) was due to the autoubiquitination of GST-Ubc2 rather than ubiquitination of endogenous lens proteins. In contrast, supplementation of lens extracts with Ubc4 or Ubc5 increased the ubiquitin conjugation activity as much as 10-fold (Fig. 3(B), compare lanes 5 and 6 with other lanes), even when the activities (judged by levels of thiol esters) of Ubc4 and Ubc5 added to the supernatant were less
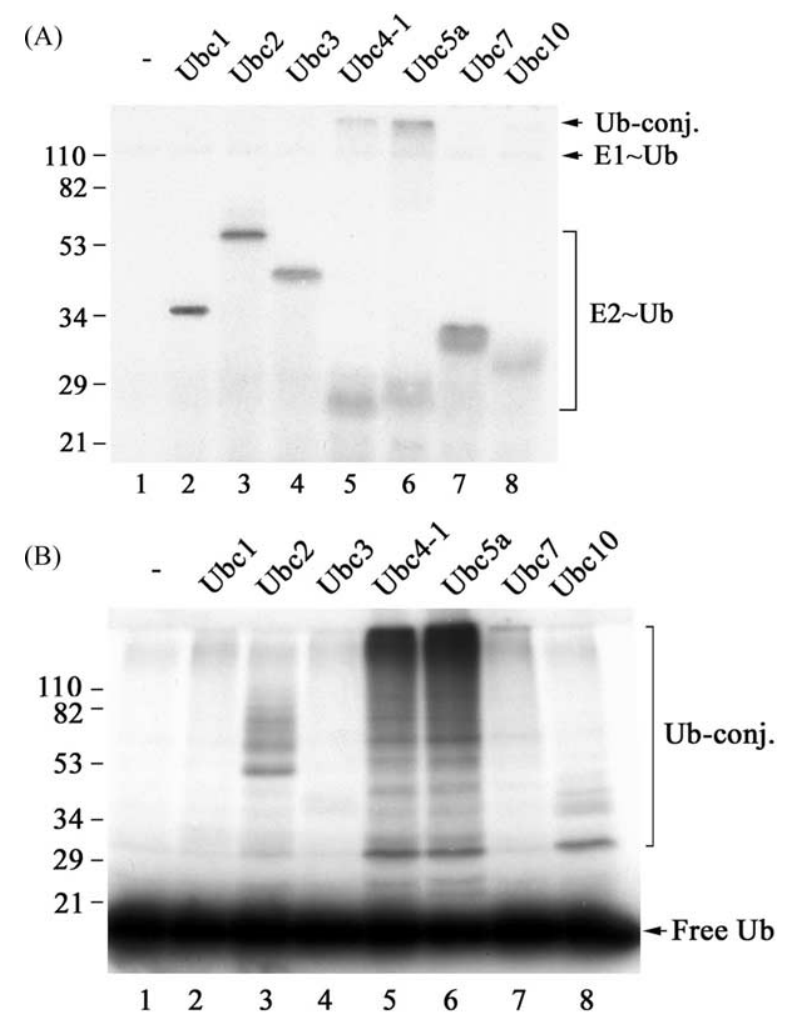

(C)

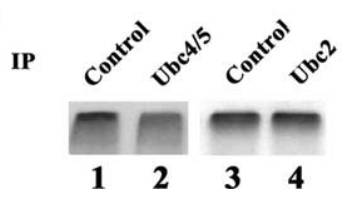

Fig. 3. Ubc4/Ubc5 are limiting in lens fibers. To test which E2 is responsible for the ubiquitination of lens proteins in lens fiber, purified E2s were added to the supernatants of lens fibers and their effect on conjugation activity was determined. Panel A showed the activity of each E2 added to the supernatants, as judged by the density of E2-ubiquitin thiol esters. Panel B showed the effects of each E2 on ubiquitin conjugation activity in supernatants of lens fibers. Panel C, effect of depletion of Ubc4/5 or Ubc2 on ubiquitin conjugation activity. Lanes 1 and 3, immunoprecipitation with control IgG; lane 2, immunoprecipitation with anti-Ubc4/5 antibody; lane 4 , immunoprecipitation with anti-Ubc2 antibody. 
than other E2s (Fig. 3(A)). Furthermore, supplementation of supernatants of the inner cortex and the nucleus of the lens with Ubc4 partially restored the conjugation activity (Fig. 4(A), compare lanes 7 and 8 with lanes 3 and 4). These results establish that limited Ubc $4 / 5$ activity in the inner regions of the lens is responsible for the decreased conjugation activity in these regions and that many other components of the UPP remain active upon maturation of lens fibers. The data also suggest that Ubc $4 / 5$ are rate-limiting with respect to conjugate formation in all regions, including the epithelial layer of the lens. The data presented in Fig. 4(A) also shows that a unique ubiquitin conjugate with molecular mass of $\sim 50 \mathrm{kDa}$ is formed in the epithelial layer and the outer cortex upon supplementation with Ubc4 (Fig. 4(A), lanes 5 and 6, pointed to by the arrow). This conjugate was only observed in the epithelial layer or in the outer cortex of calf lenses, suggesting the presence of a specific Ubc4-dependent substrate in these regions.

To corroborate that endogenous Ubc $4 / 5$ are indeed the E2s involved in the formation of ubiquitin conjugates in the lens, we immunoprecipitated Ubc $4 / 5$ from supernatants of the outer cortex of the lens and determined the ubiquitin conjugation activity in the Ubc $4 / 5$ depleted supernatant. As indicated by levels of de novo formed ubiquitin conjugates, conjugation activity was decreased by $\sim 30 \%$ in the Ubc4/5 depleted supernatants (Fig. 3(C), compare

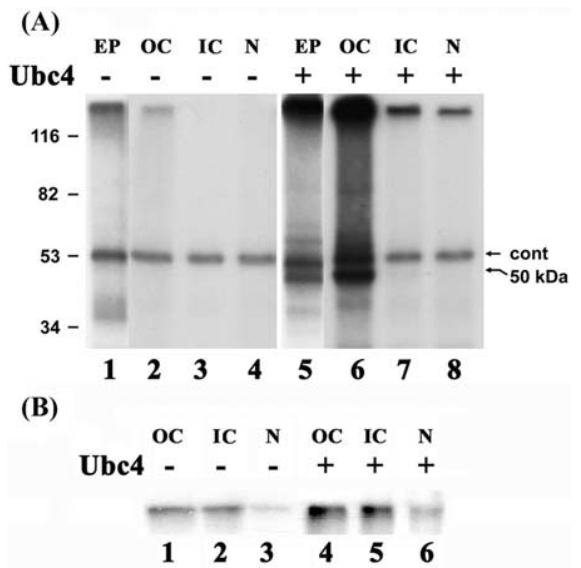

Fig. 4. Supplementation of Ubc4 partially restores the ubiquitin conjugation activity in inner regions of the lens. Young calf lenses were dissected into epithelial layer (EL), outer cortex (OC), inner cortex (IC), and nucleus (N) regions and homogenized. Ubiquitin-conjugating activity in the supernatants was determined using exogenous ${ }^{125}$ I-ubiquitin and endogenous enzymes and substrates in the presence or absence of exogenous Ubc4. Only $50 \mu \mathrm{g}$ proteins from the epithelial layer (lanes 1 and 5 of panel A) were used in each assay, whereas $500 \mu \mathrm{g}$ proteins from lens fibers (lanes 24 and 6-8 panel A, and all lanes in panel B) were used in each assay. Ubiquitin conjugation activity was monitored by the amount of high mass ${ }^{125}$ I-ubiquitin conjugates formed during incubation. Panel A, tissues from young calf lenses. Lanes 1-4, without the addition of exogenous Ubc4; lanes 5-8, with the addition of $100 \mathrm{ng}$ exogenous Ubc4. The $55 \mathrm{kDa}$ band labeled 'cont' in panel $\mathrm{A}$ is a contaminant in the ubiquitin preparation. Panel B, tissues from adult cow lens. Lanes 1-3, without additional Ubc4; lanes 4-6, with supplementation of $100 \mathrm{ng}$ exogenous Ubc4. lanes 2 with 1). In contrast, immunodepletion of Ubc2 from the lens supernatants did not affect the ubiquitin conjugation activity (Fig. 3(C), compare lane 4 with lane 3). These data indicate that endogenous Ubc $4 / 5$ are the dominant E2s for ubiquitin conjugation in the lens. The decline in levels of Ubc $4 / 5$ in inner regions of the lens appears to account for a significant proportion of the decreased ubiquitin conjugation activity in inner regions of the lens.

We previously demonstrated that lens fibers from the outer cortex of adult bovine and rat lenses have ubiquitin conjugation activity (Shang et al., 1997a,b; 1999). This experiment showed that lens fibers in the inner cortex and nuclear region of adult bovine lens also contain ubiquitin conjugation activity (Fig. 4(B)). Moreover, supplementation with Ubc4 also enhanced the ubiquitin conjugation activity (Fig. 4(B), compare lanes 4-6 with lanes 1-3). We noticed that the differences in ubiquitin conjugation activity between the outer cortex and the inner cortex of the adult bovine lens were smaller than those observed in calf lenses (compare the difference between lane 1 and lane 2 in Fig. 4(B) and that between lane 2 and lane 3 in Fig. 4(A)). This difference reflects that the outer cortex of calf lens had higher ubiquitin conjugating activity than the outer cortex of adult lens did, while the inner cortexes of adult lens and calf lens have comparable ubiquitin conjugating activity (data not shown).

\subsection{The $26 S$ proteasome remains active in lens fibers}

Ubiquitinated proteins are usually degraded by the $26 \mathrm{~S}$ proteasome. However, the activity of the $26 \mathrm{~S}$ proteasome in lens fibers could not be determined directly as we did in epithelial cells, because the large quantity of $\alpha$-crystallins, which are also substrates for the UPP (Huang et al., 1995), competitively inhibits the ubiquitination of model substrates. In order to determine the $26 \mathrm{~S}$ proteasome activity, ${ }^{125}$ I-labeled $\alpha$-lactalbumin was ubiquitinated in a proteasome-free fraction II of reticulocytes. The ubiquitinated ${ }^{125}$ I-labeled $\alpha$-lactalbumin was purified and used as the substrate for the 26S proteasome assay. As shown in Fig. 5, $\sim 48 \%$ of the ubiquitinated ${ }^{125}$ I-labeled $\alpha$-lactalbumin was degraded in supernatants of the outer cortex fibers in $60 \mathrm{~min}$ (Fig. 5, proteasome-dependent plus proteasome-independent). About $50 \%$ of the degradation was proteasomedependent (Fig. 5). Furthermore, the degradation of ubiquitinated ${ }^{125}$ I-labeled $\alpha$-lactalbumin was ATP-dependent (data not shown), indicating that it is degraded by the $26 \mathrm{~S}$ proteasome, because protein degradation mediated by the $20 \mathrm{~S}$ proteasome does not require ATP. The rates of proteasome-dependent degradation of ubiquitinated ${ }^{125}$ I-labeled $\alpha$-lactalbumin in supernatants from the inner cortex and the nucleus were comparable, which were $\sim 70 \%$ of those observed in supernatants of the outer cortex (Fig. 5). In supernatants from the lens nucleus, the proteasomedependent degradation accounts for $>80 \%$ of the degradation, and proteasome-independent degradation decreased 


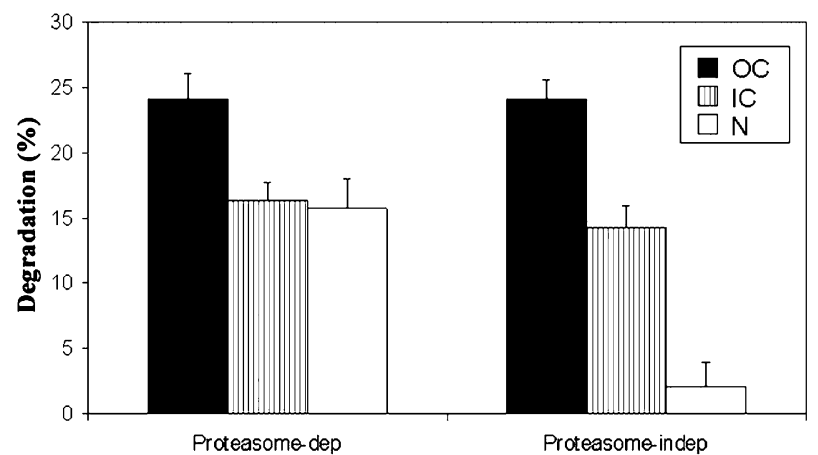

Fig. 5. All layers of lens fibers contain activity of the $26 \mathrm{~S}$ proteasome $\alpha$-Lactalbumin was labeled with ${ }^{125} \mathrm{I}$. Ubiquitin conjugates of the ${ }^{125}$ I-labeled $\alpha$-lactalbumin formed in a proteasome-free fraction from red blood cells were isolated chromatographically and were used as substrates to determine proteasome activities in each layer of lens fibers. The substrate was mixed with supernatants from each layer of the calf lens in the presence or absence of MG132, a proteasome inhibitor, for $1 \mathrm{hr}$. Degradation rates were determined as the percentage of the substrates which became TCA-soluble after incubation with lens supernatants. The portion of the degradation inhibited by MG132 was designated as proteasome-dependent degradation. OC, IC, and N represent supernatants from the outer cortex, inner cortex and the nucleus of calf lenses, respectively.

further in this region (Fig. 5). These data show that lens fibers, including those in the nuclear region, have the ability to execute ubiquitin-dependent protein degradation.

\subsection{Lens fibers contain isopeptidase activity}

Some ubiquitin conjugates are deubiquitinated by isopeptidases instead of being degraded by the proteasome. Therefore, isopeptidases act as regulators of ubiquitindependent protein degradation (Wilkinson et al. 1995; Lam et al., 1997). To determine isopeptidase activities in fiber cells, ubiquitinated ${ }^{125}$ I-labeled $\alpha$-lactalbumin was incubated with supernatants in the presence of proteasome inhibitors. Data in Fig. 6 show that a larger fraction of ubiquitinated ${ }^{125}$ I-labeled $\alpha$-lactalbumin was deconjugated upon incubation with supernatants of lens fibers. Addition of
Ubal to the reaction mixture stabilized the ubiquitinated ${ }^{125}$ I-labeled $\alpha$-lactalbumin (Fig. 6). These data showed that isopeptidases remain active in lens fibers, albeit at lower levels in the inner cortex and the nucleus of the lens (Fig. 6).

\section{Discussion}

It has been demonstrated that lens epithelial cells have a fully functional UPP (Huang et al., 1993; Shang et al. 1997a,b). We also showed that lens fibers have ubiquitin conjugation activity, and that the ubiquitin conjugation activities in both lens epithelium and fibers are up-regulated in response to oxidative stress (Shang et al. 1997a,b). In this work, we demonstrated that lens fibers, including those in the nucleus, also have a fully functional UPP. All of the components of the UPP examined in this study are present in lens fibers, although the concentrations of these components decrease from the epithelial layer to the nucleus of the lens (Fig. 1). Lens fibers in all developmental layers have the ability to form high mass ubiquitin conjugates and execute the degradation of ubiquitin conjugates by the $26 \mathrm{~S}$ proteasome. In addition, we identified that Ubc4 and Ubc5 are rate-limiting for formation of high mass ubiquitin conjugates in lens fibers (Fig. 4).

Although the decrease in ubiquitin conjugating activity in the inner regions of the lens is associated with a decrease in E1 protein levels and E1 activity, supplementation of supernatants of in inner regions of the lens with purified E1 did not restore the ubiquitin conjugation activity. In contrast, supplementation with specific E2s, Ubc4 or Ubc5, to supernatants of inner regions of the lens enhanced ubiquitin conjugation activity by $\sim 10$-fold (Fig. 4 , compare lane 7 and 8 with lane 3-4). Addition of Ubc4 to supernatants of the epithelial layer and the outer cortex also enhanced the ubiquitin conjugation activity significantly (Fig. 3(B)). However, supplementation of lens extracts with Ubc1, Ubc2, Ubc3, Ubc7 or Ubc10 had no effect on ubiquitin

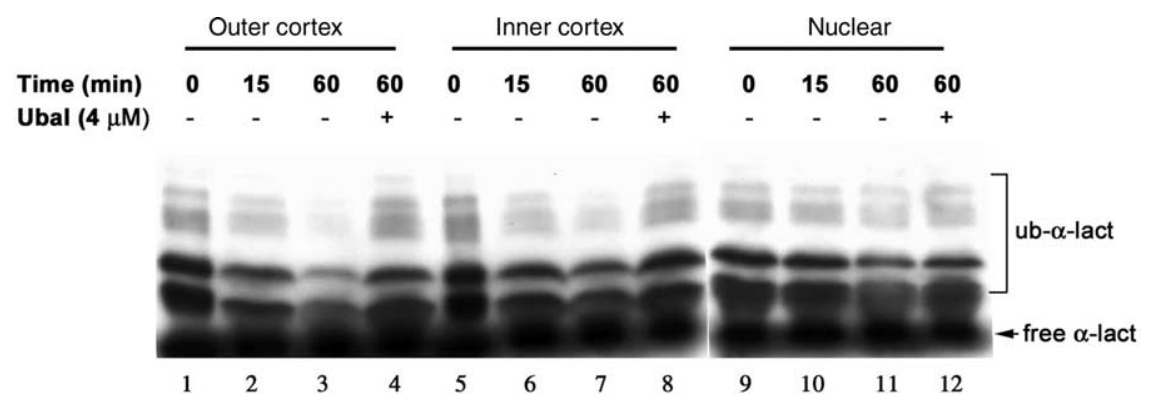

Fig. 6. Isopeptidase activity is present in all layers of lens fibers. Ubiquitin conjugates of the ${ }^{125}$ I-labeled $\alpha$-lactalbumin were prepared as in Fig. 5 and were used as substrates to determine isopeptidase activity in each layer of lens fibers. In order to prevent the degradation of ubiquitin conjugates by the $26 \mathrm{~S}$ proteasome, $80 \mu \mathrm{M}$ MG132 was present in each assay. Isopeptidase activity was measured by its ability to remove ubiquitin from the ubiquitin conjugates of ${ }^{125}$ I-labeled $\alpha$-lactalbumin. Ubiquitin aldehyde (Ubal), an isopeptidase inhibitor, was used to confirm that the disappearance of the ubiquitin conjugates during the incubation is due to isopeptidase activity. Lanes 1, 5 and 9 , ubiquitinated ${ }^{125}$ I-labeled $\alpha$-lactalbumin was incubated without lens supernatants for 60 min; lanes 2, 6 and 10, ubiquitinated ${ }^{125}$ I-labeled $\alpha$-lactalbumin was incubated with lens supernatants for 15 min; lanes 3, 7 and 11 , ubiquitinated ${ }^{125}$ I-labeled $\alpha$-lactalbumin was incubated with lens supernatants for $60 \mathrm{~min}$; lanes 4,8 and 12 , ubiquitinated ${ }^{125}$ I-labeled $\alpha$-lactalbumin were incubated with lens supernatants in the presence of $8 \mu \mathrm{M}$ Ubal for $60 \mathrm{~min}$. 
conjugation activity. These data indicate that the decreased E1 levels in the inner regions of the lens are probably not causally related to the decreased conjugation activity in these regions, but the decrease in levels of Ubc4 or Ubc5 is responsible for the decline in ubiquitin conjugation activity in the inner region of the lens. Moreover, Ubc4 and Ubc5 are rate-limiting in all regions of the lens. We previously stated that E1 is rate-limiting with respect to conjugate formation in cultured lens epithelial cells (Shang et al., 1997b). That statement should be amended to indicate that whereas E1 is limiting relative to most types of E2s and E3s, Ubc4 and Ubc5 appear to be more limiting than E1 in lens tissue.

In mammalian cells there are multiple isoforms of Ubc4 that share high sequence homology (Jensen et al., 1995; Wing and Jain, 1995). The physiological functions of some of these isoforms have been identified. For example, it has been shown that Ubc4-1 is involved in postnatal rat testis development, where it supports most of the increased protein conjugation that accompanies the developmental process (Rajapurohitam et al., 1999). Different isoforms are expressed in different stages of testis development, suggesting that they may have different roles during spermatogenesis (Wing et al., 1996; Rajapurohitam et al., 1999). It is tempting to speculate that a similar process may also be involved in lens fiber differentiation, since depletion of Ubc $4 / 5$ by immunoprecipitation reduces the conjugation activity in the outer cortex, the region most likely to contain differentiating fibers. In addition, formation of a unique $50 \mathrm{kDa}$ ubiquitin conjugate in the outer cortex upon supplementation with exogenous Ubc4 (Fig. 4(A), lanes $5-6)$ indicates a special role of Ubc4 in this region of the lens.

Ubc4 and Ubc5 are thought to interact with specific E3s that participate in recognition of specific types of substrates. The fact that addition of Ubc 4 or Ubc5 significantly restored the conjugation activity of lens fibers in vitro shows that E1, E3 and substrates for Ubc $4 / 5$ are available in all developmental regions of the lens. Typical substrates for the Ubc $4 / 5$ in yeast are damaged or obsolete proteins (Seufert and Jentsch, 1990). The substrates for Ubc $4 / 5$ in the lens may involve modified proteins, since various modifications to the long-lived lens proteins were observed in lens fibers (Chen et al., 1997; Hanson et al., 2000; Takemoto, 2001). The finding that a large portion of ubiquitin conjugation activity is ascribed to Ubc4 or Ubc5 indicates that one of the functions for the UPP in lens fibers is to degrade adversely modified or obsolete proteins. We demonstrated that the UPP was involved in degradation of oxidized proteins in lens epithelial cells (Shang et al., 2001). If the UPP has the same function in fibers as it does in the epithelial cells, the decline in the UPP activity in inner regions of the lens may contribute to the accumulation of modified or damaged proteins in these regions. The finding that supplementation of Ubc4 or Ubc5 partially restored the ubiquitin conjugation activity and presumably ubiquitin-dependent degradation capability prompts us to speculate that targeted expression of Ubc4 or Ubc5 may enhance the degradation of abnormal proteins in lens fibers and prolong the transparence of the lens.

\section{Acknowledgements}

This work is supported partially by NIH grants EY11717 (to FS), the US Department of Agriculture, under agreement No. 58-1950-9-001 (to A.T.), EY13250 (to A.T.) and Fundacao para a Ciencia e Tecnologia (FCT) Project PRAXIS/SAU/P/14197/98 (to P.P.). The authors thank Drs C.M. Pickart, K. Tanaka, and S. Wing for providing some of the precious reagents. The authors also thank Mr M. Siegal for his help in preparation of this manuscript.

\section{References}

Baumeister, W., Walz, J., Zuhl, F., Seemuller, E., 1998. The proteasome: paradigm of a self-compartmentalizing protease. Cell 92, 367-380.

Chamberlain, C.G., McAvoy, J.W., 1987. Evidence that fibroblast growth factor promotes lens fibre differentiation. Curr. Eye Res. 6, 1165-1169.

Chen, Y.C., Reid, G.E., Simpson, R.J., Truscott, R.J., 1997. Molecular evidence for the involvement of alpha crystallin in the colouration/ crosslinking of crystallins in age-related nuclear cataract. Exp. Eye Res. $65,835-840$.

Chin, D.T., Kuehl, L., Rechsteiner, M., 1982. Conjugation of ubiquitin to denatured hemoglobin is proportional to the rate of hemoglobin degradation in HeLa cells. Proc. Nat. Acad. Sci. U.S.A. 79, 5857-5861.

Ciechanover, A., Elias, S., Heller, H., Hershko, A., 1982. 'Covalent affinity' purification of ubiquitin-activating enzyme. J. Biol. Chem. 257, $2537-2542$.

Gaczynska, M., Goldberg, A.L., Tanaka, K., Hendil, K.B., Rock, K.R., 1996. Proteasome subunits X and Y alter peptidase activities in opposite ways to the interferon-g-induced subunits LMP2 and LMP7. J. Biol. Chem. 271, 17275-17280.

Hanson, S.R., Hasan, A., Smith, D.L., Smith, J.B., 2000. The major in vivo modifications of the human water-insoluble lens crystallins are disulfide bonds, deamidation, methionine oxidation and backbone cleavage. Exp. Eye Res. 71, 195-207.

Hershko, A., Ciechanover, A., 1998. The ubiquitin system. Annu. Rev. Biochem. 67, 425-479.

Hershko, A., Heller, H., Elias, S., Ciechanover, A., 1983. Components of the ubiquitin-protein ligase system. Resolution, affinity purification, and role in protein breakdown. J. Biol. Chem. 258, 8206-8214.

Huang, L.L., Jahngen-Hodge, J., Taylor, A., 1993. Bovine lens epithelial cells have a ubiquitin-dependent proteolysis system. Biochim. Biophys. Acta $1175,181-187$.

Huang, L.L., Shang, F., Nowell, T.R., Taylor, A., 1995. Degradation of differentially oxidized a-crystallins in bovine lens epithelial cells. Exp. Eye Res. 61, 45-54.

Jensen, J.P., Bates, P.W., Yang, M., Vierstra, R.D., Weissman, A.M., 1995 Identification of a family of closely related human ubiquitin conjugating enzymes. J. Biol. Chem. 270, 30408-30414.

Kuszak, J.R., 1995. The ultrastructure of epithelial and fiber cells in the crystalline lens. Int. Rev. Cytol. 163, 305-350.

Lam, Y.A., Lawson, T.G., Velayutham, M., Zweier, J.L., Pickart, C.M., 2002. A proteasomal ATPase subunit recognizes the polyubiquitin degradation signal. Nature 416, 763-767. 
Lam, Y.A., Xu, W., DeMartino, G.N., Cohen, R.E., 1997. Editing of ubiquitin conjugates by an isopeptidase in the $26 \mathrm{~S}$ proteasome. Nature 385, 737-740.

Nakamura, T., Pichel, J.G., Williams Simons, L., Westphal, H., 1995. An apoptotic defect in lens differentiation caused by human p53 is rescued by a mutant allele. Proc. Nat. Acad. Sci. U.S.A. 92 (13), 6142-6146.

Obin, M., Nowell, T., Taylor, A., 1994. The photoreceptor G-protein transducin $(\mathrm{Gt})$ is a substrate for ubiquitin dependent proteolysis. Biochem. Biophys. Res. Commun. 200, 1169-1176.

Pan, H., Griep, A.E., 1994. Altered cell cycle regulation in the lens of HPV-16 E6 or E7 transgenic mice: implications for tumor suppressor gene function in development. Genes Dev. 8, 1285-1299.

Rajapurohitam, V., Morales, C.R., El-Alfy, M., Lefrançois, S., Bedard, N., Wing, S., 1999. Activation of UBC4-dependent pathway of ubiquitin conjugation during postnatal development of the rat testis. Dev. Biol. 212, 217-228.

Seufert, W., Jentsch, S., 1990. Ubiquitin-conjugating enzymes UBC4 and UBC5 mediate selective degradation of short-lived and abnormal proteins. Embo J. 9, 543-550.

Shang, F., Deng, G., Obin, M., Wu, C.C., Gong, X., Smith, D., Laursen, R.A., Andley, U.P., Reddan, J.R., Taylor, A., 2001. Ubiquitinactivating enzyme (E1) isoforms in lens epithelial cells: origin of translation, E2 specificity and cellular localization determined with novel site-specific antibodies. Exp. Eye Res. 73, 827-836.

Shang, F., Gong, G., McAvoy, J.W., Chamberlain, C., Nowell, T.R., Taylor, A., 1999. Ubiquitin-dependent pathway is up-regulated in differentiating lens cells. Exp. Eye Res. 68, 179-192.
Shang, F., Gong, X., Palmer, H.J., Nowell, T., Taylor, A., 1997a. Age-related decline in ubiquitin conjugation in response to oxidative stress in the lens. Exp. Eye Res. 64, 21-30.

Shang, F., Gong, X., Taylor, A., 1997b. Activity of ubiquitin dependent pathway in response to oxidative stress: ubiquitin activating enzyme (E1) is transiently upregulated. J. Biol. Chem. 272, 23086-23093.

Shang, F., Nowell Jr, T.R., Taylor, A., 2001. Removal of oxidatively damaged proteins from lens cells by the ubiquitin-proteasome pathway. Exp. Eye Res. 73, 229-238.

Shang, F., Taylor, A., 1995. Oxidative stress and recovery from oxidative stress are associated with altered ubiquitin conjugating and proteolytic activities in bovine lens epithelial cells. Biochem. J. 307, 297-303.

Takemoto, L., 2001. Deamidation of Asn-143 of gamma S crystallin from protein aggregates of the human lens. Curr. Eye Res. 22, 148-153.

Tanaka, K., Tsurumi, C., 1997. The 26S proteasome: subunits and functions. Mol. Biol. Rep. 24, 3-11.

Wilkinson, K.D., Tashayev, V.L., O’Connor, L.B., Larsen, C.N., Kasperek, E., Pickart, C.M., 1995. Metabolism of the polyubiquitin degradation signal: structure, mechanism, and role of isopeptidase T. Biochemistry 34, 14535-14546.

Wing, S.S., Bedard, N., Morales, C., Hingamp, P., Trasler, J., 1996. A novel rat homologue of the S. Cerevisiae ubiquitin conjugating enzyme UBC4 with distinct biochemical features is induced during spermatogenesis. Mol. Cell. Biol. 16, 4064-4072.

Wing, S., Jain, P., 1995. Molecular cloning, expression, and characterization of a ubiquitin conjugation enzyme (E2 $17 \mathrm{~KB})$ highly expressed in rat testis. Biochem. J. 305, 125-132. 